\title{
Integrable systems connected with black holes
}

\author{
H. Demirchian* \\ Ambartsumian Byurakan Astrophysical Observatory, Byurakan, 0213, Armenia
}

\begin{abstract}
We studied some important questions in general relativity and mathematical physics mainly related to the two most important solutions of the theory of relativity - gravitational waves and black holes. In particular, the work is related to astrophysical shock waves, gravitational waves, black holes, integrable systems associated with them as well as their quantum equivalents. We studied the effects of null shells on geodesic congruences and suggested a general covariant definition of the gravitational memory effect. Thus, we studied observable effects that astrophysical shock waves can have on test particles after cataclysmic astrophysical events. We studied the geodesics of massive particles in Near Horizon Extremal Myers-Perry (NHEMP) black hole geometries. This is the space-time in the vicinity of the horizon of higher dimensional rotating black holes. Thus, this work can have applications for studying accretions of black holes. The system is also important in mathematical physics as it describes integrable (in special cases superintegrable) system, where the constants of motion are fully studied. On the other hand, the quantum counterparts of this and other integrable systems are studied as well and a new technique is suggested for geometrization of these systems.
\end{abstract}

Keywords: shock wave, impulsive signal, gravitational memory effect, black hole, integrable system, Klein-Gordon equation, geometrization

\section{Introduction}

This work is devoted to the study of some important questions in general relativity. They include topics related to astrophysical shock waves, impulsive signals, gravitational memory effect, black hole geometries and integrable systems connected with them.

We begin by studying the effects that an impulsive signal in a singular hypersurface can have on a particle which encounters it. We propose a new approach for studying the effect of null shells on null geodesic congruences. This is an exact method which allows one to easily calculate the change in the expansion, shear and rotation of the congruence upon crossing the shell and its evolution to the future of the shell. We find that the effect of the shell on the congruence is a discontinuity in the B-tensor (the gradient of the geodesic vector). We call this the B-memory effect, which is a more covariant way of describing the gravitational memory effect.

Furthermore, we study Hamilton-Jacobi system of a particle in Near Horizon geometry of Extremal Myers-Perry (NHEMP) black hole. The question of integrability of special cases of fully non-isotropic and fully isotropic cases is addressed in this description. The general case, when groups of equal and non-equal rotation parameters exist, is studied. It is shown that this problem reduces to its special cases of fully non-isotropic and fully isotropic NHEMP conformal mechanics. At the end we turn to the integrability problem of the Hamilton-Jacobi system in so-called Near Horizon Extremal Vanishing Horizon Myers-Perry black hole (NHEVHMP).

We have also studied quantum aspects of the superintegrable systems encountered in black hole backgrounds. We propose a geometrization procedure which associates to a non-relativistic quantum particle in a potential on a curved spacetime a purely geodesic motion in another geometry. In other words, we propose a correspondence between the solutions of Schroedinger equation and KleinGordon equation. We explain this procedure on the example of the Higgs oscillator and superintegrable Rosochatius system.

*demhov@bao.sci.am 


\section{Geodesic congruences, impulsive gravitational waves and gravita- tional memory}

The study of impulsive gravitational waves in the form of null shells has recently received renewed attention due to their possible role in the transfer of information from black hole horizons to null infinity. As the black hole horizon is a killing horizon, there is an infinite variety of ways to attach (solder) the black hole interior to the black hole exterior creating a null shell on the horizon (Barrabes and Israel, 1991, Blau and O'Loughlin, 2016). A subclass of these can be shown to correspond to BMS like supertranslations. Furthermore the long studied BMS supertranslations at null infinity of asymptotically flat spaces are linked to the physics of soft gravitons which appear to play an important role in restoring information not seen in the hard gravitons of Hawking radiation (Hawking et al., 2017, 2016). In turn the soft gravitons are related to the gravitational memory effect (Strominger and Zhiboedov, 2016).

Gravitational memory (Zel'dovich and Polnarev, 1974, Strominger, 2017, Ashtekar et al., 2018) is the classical change in nearby geodesics in an asymptotically flat region of space-time as they pass through an outgoing gravitational wave. The study of the effect of a null shell on a time-like congruence that crosses it has been addressed by Barrabes and Hogan (Barrabès and Hogan, 2003, Barrabes and Hogan, 2001). They calculated the change in the tangent vector and the geodesic deviation vector together with the expansion, shear and rotation upon crossing an impulsive gravitational wave and found a jump in the acceleration of the geodesic and derivatives of the geodesic deviation vector proportional to the stress-energy content and gravitational wave components of the shell.

To further understand the relationship between gravitons and gravitational memory it is thus important to study the effect of waves on null geodesic congruences, not only as the congruence crosses the wave but also the future evolution of the congruence.

We have presented (O'Loughlin and Demirchian, 2019) a new approach for studying congruences that cross a singular hypersurface. Our method is based on the physically justified assumption that the geodesic vector of a test particle is continuous across the hypersurface when using continuous coordinates. To obtain the geodesic flow to the future of the hypersurface one simply needs to do a coordinate transformation on the past coordinates to go to a continuous coordinate system. The resulting transformation on the geodesic congruence in $\mathcal{M}^{-}$gives initial conditions on $\mathcal{N}$ to develop the geodesic vector field on $\mathcal{M}^{+}$to the future.

We then proved that a parallel congruence upon crossing the shell gives rise to a hypersurface orthogonal congruence to the future of the shell, and in particular that the shell gives rise to a discontinuity in the B-tensor of the congruence. In general the jump in the expansion is determined by the energy density and currents on the shell while the jump in the shear is determined by the gravitational wave component together with the surface currents. Although we derived these results using a particular congruence, it is clear that the results are independent of the choice of congruence in the case of BMS supertranslations for which the surface currents are zero. We also provide a general argument that a hypersurface orthogonal congruence before the shell will give rise to a hypersurface orthogonal congruence to the future.

The change in the B-tensor after the passage of an outgoing gravitational wave leads to a covariant description of the gravitational memory effect - the B-memory effect.

\section{Integrability of geodesics in near-horizon extremal Myers-Perry black holes}

The system is called integrable if in a dynamical system with $N$ degrees of freedom ( $2 N$ dimensional phase space), the number of independent symmetries is equal to $N$. If the system possesses $N+p$, $1 \leq p \leq N-1$, independent symmetries (and hence functionally independent constants of motion), it is called superintegrable.

The question of integrability of particle dynamics (Hamilton-Jaconi system) on black hole or near horizon geometries have been extensively analyzed in the literature e.g. see (Carter, 1968, Galajinsky, 
2013, Bellucci et al., 2012, Saghatelian, 2012, Galajinsky et al., 2013, Frolov et al., 2007). In particular, it has been shown that the problem is (super)integrable for a large class of black holes.

Given an extremal black hole there are general theorems stating that in the near horizon limit we obtain a usually smooth geometry with larger isometry group than the original extremal black hole. It is hence an interesting question to explore if this symmetry enhancement yields further independent constants of motion and how it affects the integrability of particle dynamics. This question, besides the academic interests, is also relevant to some of the observations related to black holes: It is now a well-accepted fact that there are fast rotating black holes in the sky which are well modeled by an extreme Kerr geometry and the matter moving around these black holes in their accretion disks are essentially probing the near horizon geometry.

The isometry group of generic stationary extremal black holes in the near horizon region is shown to have an $S O(2,1)=S L(2, \mathbb{R})$ part. Therefore, particle dynamics on the near horizon extreme geometries possesses dynamical $0+1$ dimensional conformal symmetry, i.e. it defines a "conformal mechanics" (Galajinsky, 2013, Bellucci et al., 2012, Saghatelian, 2012, Galajinsky et al., 2013). This allows to reduce the problem to the study of system depending on latitudinal and azimuthal coordinates and their conjugate momenta with the effective Hamiltonian being Casimir of conformal algebra. Such associated systems have been investigated from various viewpoints and have been named "angular or spherical mechanics".

We analyzed massive and massless geodesics in the Near Horizon Extremal Myers-Perry background (Demirchian et al., 2018a, Demirchyan et al., 2018, Demirchian et al., 2018b, Demirchian, 2017). We started with a system with $2 N+1+\sigma$ variables. Fixing the momenta associated with the isometries, we obtained and focused the $N-1+\sigma$ dimensional "angular mechanics" part. In this sector, whenever $N$ number of rotation parameters $m_{i}$ of the background metric are equal the $U(1)^{N}$ isometry is enhanced to $U(N)$ and this latter brings about other second rank Killing tensors. All in all, in the fully isotropic case in odd dimensions with $U\left(\frac{d-1}{2}\right)$ isometry, the $d-2$ dimensional spherical mechanics part is maximally superintegrable, it has $N+(N-2)=2 N-2$ extra constants of motion. The fully isotropic case in even dimensions, however, is not maximally superintegrable; it has still $2 N-1$ extra Killing tensors (one less than the $N$ constants of motion to make the system fully superintegrable).

We also discussed the Extremal Vanishing Horizon (EVH) case, which derives from odd dimensional extremal MP when one of the rotation parameters $a_{i}$ vanishes. In the general NHEVHMP case, where the background isometry is $S O(2,2) \times U(1)^{\frac{d-3}{2}}$ the number of independent charges associated with Killing vectors is $\frac{d+1}{2}$. Despite enhancement of the isometry group compared to the generic NHEMP case, we found that this symmetry enhancement does not add independent constants of motion, the system in general does not poses extra constants of motion and remains just integrable.

\section{Superintegrable quantum systems and resonant spacetimes}

Geometrization of dynamics is a recurrent theme in theoretical physics. While it has underlied such fundamental developments as the creation of General Relativity and search for unified theories of interactions, it also has a more modest (but often fruitful) aspect of reformulating conventional, wellestablished theories in more geometrical terms, in hope of elucidating their structure. One particular approach of the latter type is the Jacobi metric. This energy-dependent metric simply encodes as its geodesics the classical orbits of a nonrelativistic mechanical particle on a manifold moving in a potential.

We have presented (Evnin et al., 2018) a procedure associating to quantum systems a Klein-Gordon equation on a static spacetime. For systems with the quadratic energy spectrum, our procedure results in spacetimes with a resonant spectrum of evenly spaced frequencies. This correspondence generalizes the previously known relation between the Higgs oscillator and (global) Anti-de Sitter spacetime.

Implementing our procedure in practice requires solving a nonlinear elliptic equation. The latter form is closely reminiscent of elliptic equations extensively studied in relation to classic 'prescribed scalar curvature' problems of differential geometry (though the exact power appearing in the power-law nonlinearity is different). If one aims at constructing a massless Klein-Gordon (i.e., wave) equation 
corresponding to the original quantum-mechanical system, the nonlinearity drops out, resulting in a much simpler problem. In this case, known ground state wavefunctions for the original quantum system can be utilized for the conversion procedure. We have demonstrated how this approach works for superintegrable Rosochatius systems, resulting in a family of spacetimes resonant with respect to the massless wave equation.

\section{Summary}

Here we outline of the main results of this thesis.

- A new approach has been suggested for studying the effects of impulsive gravitational waves on congruences encountering them. The technique has been applied on null congruences. It has been established that hypersurface orthogonal null congruences stay such after crossing the shell.

- A covariant definition of the gravitational memory effect has been suggested based on the Btensor of the congruence. The relations between the components of the B-tensor and the stressenergy tensor of the shell have been derived. The B-tensor has been calculated and the approach has been demonstrated for BMS type soldering.

- A common description has been introduced for even and odd dimensional NHEMP geometries. This description was used to prove that the even dimensional fully non-isotropic NHEMP system is integrable.

- Integrals of motion, as well as the Killing vectors of the fully non-isotropic NHEMP in arbitrary dimensions have been presented in initial coordinates. We found a non-trivial transformation between the integrals of motion of fully non-isotropic and fully isotropic NHEMP black hole geometries.

- We separated the variables of the most general partially isotropic NHEMP and showed its transformation to the special cases of fully non-isotropic and isotropic NHEMP.

- A new approach has been suggested for mapping Schrödinger equation on a curved background to a Klein-Gordon equation on the background of another geometry. We have shown that this procedure greatly simplifies for systems with quadratic spectra and applied it on the Higgs oscillator and the superintegrable Rosochatius system.

\section{References}

C. Barrabes and W. Israel. Thin shells in general relativity and cosmology: The Lightlike limit. Phys. Rev., D43:1129-1142, 1991. doi: 10.1103/PhysRevD.43.1129.

Matthias Blau and Martin O'Loughlin. Horizon Shells and BMS-like Soldering Transformations. JHEP, 03:029, 2016. doi: 10.1007/JHEP03(2016)029.

Stephen W. Hawking, Malcolm J. Perry, and Andrew Strominger. Superrotation Charge and Supertranslation Hair on Black Holes. JHEP, 05:161, 2017. doi: 10.1007/JHEP05(2017)161.

Stephen W. Hawking, Malcolm J. Perry, and Andrew Strominger. Soft Hair on Black Holes. Phys. Rev. Lett., 116(23):231301, 2016. doi: 10.1103/PhysRevLett.116.231301.

Andrew Strominger and Alexander Zhiboedov. Gravitational Memory, BMS Supertranslations and Soft Theorems. JHEP, 01:086, 2016. doi: 10.1007/JHEP01(2016)086.

Y. B. Zel'dovich and A. G. Polnarev. Radiation of gravitational waves by a cluster of superdense stars. Soviet Astronomy, 18:17, August 1974.

Andrew Strominger. Lectures on the Infrared Structure of Gravity and Gauge Theory. 2017. 
Abhay Ashtekar, Miguel Campiglia, and Alok Laddha. Null infinity, the BMS group and infrared issues. Gen. Rel. Grav., 50(11):140-163, 2018. doi: 10.1007/s10714-018-2464-3.

C. Barrabès and P. A. Hogan. Singular Null Hypersurfaces in General Relativity: Light-Like Signals from Violent Astrophysical Events. World Scientific Publishing Co, 2003. doi: 10.1142/5454.

C. Barrabes and P. A. Hogan. Detection of impulsive light - like signals in general relativity. Int. J. Mod. Phys., D10:711-722, 2001. doi: 10.1142/S0218271801001098.

Martin O'Loughlin and Hovhannes Demirchian. Geodesic congruences, impulsive gravitational waves and gravitational memory. Phys. Rev., D99(2):024031, 2019. doi: 10.1103/PhysRevD.99.024031.

B. Carter. Hamilton-Jacobi and Schrodinger separable solutions of Einstein's equations. Commun. Math. Phys., 10(4):280-310, 1968. doi: 10.1007/BF03399503.

Anton Galajinsky. Near horizon black holes in diverse dimensions and integrable models. Phys. Rev., D87(2):024023, 2013. doi: 10.1103/PhysRevD.87.024023.

Stefano Bellucci, Armen Nersessian, and Vahagn Yeghikyan. Action-Angle Variables for the Particle Near Extreme Kerr Throat. Mod. Phys. Lett., A27:1250191, 2012. doi: 10.1142/S021773231250191X.

Armen Saghatelian. Near-horizon dynamics of particle in extreme Reissner-Nordstróm and ClementGal'tsov black hole backgrounds: action-angle variables. Class. Quant. Grav., 29:245018, 2012. doi: 10.1088/0264-9381/29/24/245018.

Anton Galajinsky, Armen Nersessian, and Armen Saghatelian. Superintegrable models related to near horizon extremal Myers-Perry black hole in arbitrary dimension. JHEP, 06:002, 2013. doi: 10.1007/JHEP06(2013)002.

Valeri P. Frolov, Pavel Krtous, and David Kubiznak. Separability of Hamilton-Jacobi and KleinGordon Equations in General Kerr-NUT-AdS Spacetimes. JHEP, 02:005, 2007. doi: 10.1088/ 1126-6708/2007/02/005.

Hovhannes Demirchian, Armen Nersessian, Saeedeh Sadeghian, and M. M. Sheikh-Jabbari. Integrability of geodesics in near-horizon extremal geometries: Case of Myers-Perry black holes in arbitrary dimensions. Phys. Rev., D97(10):104004, 2018a. doi: 10.1103/PhysRevD.97.104004.

H. Demirchyan, A. Nersessian, S. Sadeghian, and M. M. Sheikh-Jabbari. Integrability of Geodesics in Near-Horizon Extremal Vanishing Horizon Myers-Perry Black Holes. Phys. Atom. Nucl., 81(6): 907-911, 2018. doi: 10.1134/S106377881806011X.

H. Demirchian, T. Hakobyan, A. Nersessian, and M. M. Sheikh-Jabbari. Myers-Perry Conformal Mechanics. Phys. Part. Nucl., 49(5):860-864, 2018b. doi: 10.1134/S1063779618050167.

Hovhannes Demirchian. Note on constants of motion in conformal mechanics associated with near horizon extremal Myers-Perry black holes. Mod. Phys. Lett., A32(27):1750144, 2017. doi: 10.1142/ S0217732317501449.

Oleg Evnin, Hovhannes Demirchian, and Armen Nersessian. Mapping superintegrable quantum mechanics to resonant spacetimes. Phys. Rev., D97(2):025014, 2018. doi: 10.1103/PhysRevD.97. 025014 . 